\title{
Multi-symplectic Birkhoffian Structure for PDEs with Dissipation Terms*
}

\author{
Hongling $\mathrm{Su} \quad$ Mengzhao Qin \\ CAST (World Laboratory), \\ Institute of Computational Mathematics \\ and Scientific/Engineering Computing, \\ Academy of Mathematics and System Sciences, \\ Chinese Academy of Sciences, Beijing, 100080, China.
}

November 2, 2018

\begin{abstract}
The multi-symplectic form for Hamiltonian PDEs leads to a general framework for geometric numerical schemes that preserve a discrete version of the conservation of symplecticity. The cases for systems or PDEs with dissipation terms has never been extended. In this paper, we suggest a new extension for generalizing the multi-symplectic form for Hamiltonian systems to systems with dissipation which never have remarkable energy and momentum conservation properties. The central idea is that the PDEs is of a first-order type that has a symplectic structure depended explicitly on time variable, and decomposed into distinct components representing space and time directions. This suggest a natural definition of multi-symplectic Birkhoff's equation as a multi-symplectic structure from that a multi-symplectic dissipation law is constructed. We show that this definition leads to deeper understanding relationship between functional principle and PDEs. The concept of multi-symplectic integrator is also discussed.
\end{abstract}

\section{Introduction}

The multi-symplectic Hamilton's Equation is proposed first time by Bridges in 1997[1]. Birkhoff's Equation is named by Santilli[2, who just discussed 1dimensional cases. We return to multi-symplectic Hamilton's Equation, and we just discuss 1+1-dimensional cases. It is a local concept of symplecticity

\footnotetext{
* Supported by the Special Funds for Major State Basic Research Projects , G 1999, 032800
} 
for Hamiltonian systems. The concept can be abstracted as follows. Let $M$ and $K$ be any skew symmetric matrices on $\mathbb{R}^{n}(n \geq 3)$ and Let $S: \quad \mathbb{R}^{n} \longrightarrow$ $\mathbb{R}$ be any smooth function. Then we will call a system of the following form a Hamiltonian system on a multi-symplectic structure,

$$
M z_{t}+K z_{x}=\nabla_{z} S(z, x, t), \quad z \in \mathbb{R}^{n}, \quad(x, t) \in \mathbb{R}^{2},
$$

where the general gradient $\nabla_{z}$ is defined with respect to the standard inner product on $\mathbb{R}^{n}$, denoted by $\langle\cdot, \cdot\rangle$. Associated $M$ and $K$ are the so-called pre-symplectic forms

$$
\omega(U, V)=<M U, V>, \quad \kappa(U, V)=<K U, V>.
$$

The two 2-forms $\omega$ and $\kappa$ define two symplectic structure on $\mathbb{R}^{m} \quad(m=$ $\operatorname{rank} M \leq n)$ and $\mathbb{R}^{k} \quad(k=\operatorname{rank} K \leq n)$ respectively. The pair $(\omega, \kappa)$ satisfies conservation of symplecticity

$$
\frac{\partial}{\partial t} \omega+\frac{\partial}{\partial x} \kappa=0
$$

A multi-symplectic integrator is numerical approximation of equation (11)

$$
M \partial t^{i, j} z_{i, j}+K \partial x^{i, j} z_{i, j}=\left(\nabla_{z} S\left(z_{i, j}, x_{i}, t_{j}\right)\right)_{i, j}, \quad \text { where } \quad z_{i, j}=z\left(x_{i}, t_{j}\right)
$$

which has a discrete conservation law as

$$
\partial t^{i, j} \omega_{i, j}+\partial x^{i, j} \kappa_{i, j}=0
$$

The problem with this type to abstract and to discrete the systems or PDEs is that it is subject to conservative systems or nonconservative systems without dissipation terms. A more general multi-symplectic structure is to represent some nonconservative systems with dissipation terms, since,- different from form (11) - symplecticity may depend on time variables explicitly, and the right hand is a gradient function (vector function) plus a dissipation terms.

Similarly, let $M(z, x, t)$ and $K(z, x, t)$ are skew symmetric metrics on $\mathbb{R}^{n} \geq 2$, and $B ; \quad \mathbb{R}^{n} \times \mathbb{R}^{2} \longrightarrow \mathbb{R}$ be any smooth function. Then a Birkhoffian system under the following multi-symplectic structure is given

$$
M(z, x, t) z_{t}+K(z, x, t) z_{x}=\nabla_{z} B(z, x, t)+\frac{\partial F}{\partial t}+\frac{\partial G}{\partial x},
$$


where $M$ and $F, K$ and $G$ are independent. We will show their relationship in the next section.

Structure (6) is interesting for two reasons: it is a natural generalization of structure (11) as well as Birkhoff's representation for ODEs to PDEs, and it can be used to develop symplectic numerical integrators for nonconservative systems with dissipation terms. For example, consider the equation describe a linear damped string

$$
u_{t t}-u_{x x}+u+\alpha u_{t}+\beta u_{x}=0 .
$$

we introduce the new variables $p$ and $q$, defined below, and equation (7) is rewritten as first-order form

$$
u_{t}=p, \quad u_{x}=q, \quad p_{t}-q_{x}+u+2 p=0,
$$

or, with $z=(u, p, q)^{\mathrm{T}} \in \mathbb{R}^{3}$, we obtain a multi-symplectic Birkhoff's equations (ds1.6), where

$$
M=\left(\begin{array}{ccc}
0 & \mathrm{e}^{\alpha t-\beta x} & 0 \\
-\mathrm{e}^{\alpha t-\beta x} & 0 & 0 \\
0 & 0 & 0
\end{array}\right), \quad K=\left(\begin{array}{ccc}
0 & 0 & -\mathrm{e}^{\alpha t-\beta x} \\
0 & 0 & 0 \\
\mathrm{e}^{\alpha t-\beta x} & 0 & 0
\end{array}\right) \text {, }
$$

and

$$
\begin{array}{r}
\left.F=\left(-\frac{1}{2} e^{\alpha t-\beta x} p, \frac{1}{2} e^{\alpha t-\beta x} u, 0\right)^{\mathrm{T}}, 0\right), \\
\left.G=\left(\frac{1}{2} e^{\alpha t-\beta x} q, 0, \frac{1}{2} e^{\alpha t-\beta x} u, 0\right)^{\mathrm{T}}\right), \\
B=-\frac{1}{2} \mathrm{e}^{\alpha t-\beta x}\left[u^{2}+p^{2}-q^{2}+\alpha u p+\beta u q\right] .
\end{array}
$$

We now show an outline of the paper. In section 2 we introduce accurate definition of multi-symplectic Birkhoff's equations. From the point of inverse variational problem we discuss the conditions of self-adjointness for Birkhoff's equations. This leads naturally to a variational principle. In section 3, we introduce continuous and discrete dissipation laws of symplecticity, energy and momentum, then give definition of multi-symplectic Birkhoffian integrator. In section 4,we consider equation (7) to illustrate how a multi-symplectic Birkhoffian integrator to preserve the discrete version of the dissipation laws. 


\section{Multi-symplectic Birkhoff's Equation}

The general multi-symplectic structure we shall study is given by

$$
\begin{aligned}
& {\left[\frac{\partial F_{\nu}(z, x, t)}{\partial z^{\mu}}-\frac{\partial F_{\mu}(z, x, t)}{\partial z^{\nu}}\right] \frac{\partial z_{\nu}}{\partial t}+\left[\frac{\partial G_{\nu}(z, x, t)}{\partial z^{\mu}}-\frac{\partial G_{\mu}(z, x, t)}{\partial z^{\nu}}\right] \frac{\partial z_{\nu}}{\partial x}} \\
& -\left[\frac{\partial B(z, x, t)}{\partial z^{\mu}}+\frac{\partial F_{\mu}(z, x, t)}{\partial t}+\frac{\partial G_{\mu}(z, x, t)}{\partial x}\right]=0 .
\end{aligned}
$$

Equations (11) are called multi-symplectic Birkhoff's equations. Why using this name is reviewed in Ref. [2]. The function $B(z, x, t)$ is called Birkhoffian. A representation of a system via multi-symplectic Birkhoff's equations is called a multi-symplectic Birkhoffian representation, and the system is called multi-symplectic system.

Now we consider a general first-order form in the self-explanatory unified notation

$$
M_{\mu \nu}(z, x, t) z_{t}^{\nu}+K_{\mu \nu} z_{x}^{\nu}+D_{m} u(z, x, t)=0 .
$$

We give the conditions of variational self-adjointness for equation (12) as follows.

Theorem 2.1. (self-adjointness of the covariant general form) A necessary and sufficient condition for equations (11) to be self-adjoint in a region $\widetilde{\Re}$ of points $(z, x, t)$ is that all the following conditions

$$
\begin{gathered}
M_{\mu \nu}+M_{\nu \mu}=0, \quad K_{\mu \nu}+K_{\nu \mu}=0, \\
\frac{\partial M_{\mu \nu}}{\partial z^{\tau}}+\frac{\partial M_{\nu \tau}}{\partial z^{\mu}}+\frac{\partial M_{\tau \mu}}{\partial z^{\nu}}, \quad \frac{\partial K_{\mu \nu}}{\partial z^{\tau}}+\frac{\partial K_{\nu \tau}}{\partial z^{\mu}}+\frac{\partial K_{\tau \mu}}{\partial z^{\nu}}, \\
\frac{\partial M_{\mu \nu}}{\partial t}=\frac{\partial D}{\partial z^{\nu}}-\frac{\partial D}{\partial z^{\mu}},
\end{gathered}
$$

are identically verified in $\widetilde{\Re}$.

Note that the conditions of self-adjointness are formulated for the covariant form because this is the form derivable from a variational principle which is the most general possible first-order action functional given by the pfaffian action

$$
£(\widetilde{E})=\int_{t_{1}}^{t_{2}} \int_{x_{1}}^{x_{2}} \mathrm{~d} t \mathrm{~d} x\left[F \nu(z, x, t) z_{t}^{\nu}+G_{\nu}(z, x, t) z_{x}^{\nu}-B(z, x, t)\right](\widetilde{E}) .
$$


Strip away the integral from $£(\widetilde{E})$ in (14), define a general Lagrange density $\mathcal{L}=F \nu(z, x, t) z_{t}^{\nu}+G_{\nu}(z, x, t) z_{x}^{\nu}-B(z, x, t)$. It doesn't exist a Legendre transformation (including conjugate momenta ) leads reduction from the Lagrange equation to Hamilton's equation. While from the variational principle we can write a Birkhoff's equation directly, and for any self-adjoint covariant system, a variational principle can be constructed.

Proposition 2.1. (Self-adjointness of Birkhoff's Equations). Necessary and sufficient condition for a general first order system (ds2.2) to be selfadjoint is that it is of the Birkhoffian type, i.e.,

$$
\begin{aligned}
& M_{\mu \nu}(z, x, t) z_{t}^{\nu}+K_{\mu \nu} z_{x}^{\nu}+D_{m} u(z, x, t)= \\
& {\left[\frac{\partial F_{\nu}(z, x, t)}{\partial z^{\mu}}-\frac{\partial F_{\mu}(z, x, t)}{\partial z^{\nu}}\right] \frac{\partial z_{\nu}}{\partial t}+\left[\frac{\partial G_{\nu}(z, x, t)}{\partial z^{\mu}}-\frac{\partial G_{\mu}(z, x, t)}{\partial z^{\nu}}\right] \frac{\partial z_{\nu}}{\partial x}} \\
& -\left[\frac{\partial B(z, x, t)}{\partial z^{\mu}}+\frac{\partial F_{\mu}(z, x, t)}{\partial t}+\frac{\partial G_{\mu}(z, x, t)}{\partial x}\right]=0 .
\end{aligned}
$$

\section{Multi-symplecticity Dissipation Laws and Multi- symplectic Integrators}

The multi-symplectic Birkhoff's equations (11) determines 2 two-forms $\omega$ and kappa. Since

$$
\begin{gathered}
\frac{\partial}{\partial t} \omega(U, V)=<M U_{t}, V>+<M U, V_{t}>+<M_{t} U, V>, \\
\frac{\partial}{\partial x} \kappa(U, V)=<K U_{x}, V>+<K U, V_{x}>+<K_{x} U, V>,
\end{gathered}
$$

one obtains

$$
\begin{aligned}
\frac{\partial}{\partial t} \omega(U, V)+\frac{\partial}{\partial x} \kappa(U, V) & =<M U_{t}+K U_{x}, V>-<U, M V_{t}+K V_{x}>+<M_{t} U+K_{x} U, V> \\
& =<F_{z t} U+G_{z x} U, V>-<F_{z t} V+G_{z x} V, U> \\
& =0 .
\end{aligned}
$$

This statement is true for any solution pair $(U, V)$ and we write is as

$$
\partial_{t} \omega+\partial_{x} \kappa=0 .
$$

This is different from the conservation law (3), - although they look like each other-, since that $M_{t} \neq 0$ and $K_{x} \neq 0$. Therefore we define this law 
as a multi-symplectic dissipation law for Birkhoffian system, and in the next section this definition will be verified very natural.

The numerical framework is start from the Birkhoff's equation (11) with the associated dissipation law of symplecticity (18). The idea is to develop a numerical scheme to approximate equations (11) and it also preserve a discrete version of dissipation of symplecticity. A numerical discretization of (18) can be written schematically as

$$
\begin{aligned}
M_{i, j} \partial_{t}^{i, j} z_{i, j}+K_{i, j} \partial_{x}^{i, j} z_{i, j} & =\left(\nabla_{z} S\left(z_{i, j}, x_{i}, t_{j}\right)\right)_{i, j}+\left(\partial_{t} F\left(z_{i, j}, x_{i}, t_{j}\right)\right)_{i, j} \\
& +\left(\partial_{x} G\left(z_{i, j}, x_{i}, t_{j}\right)\right)_{i, j}, \quad z_{i, j}=z\left(x_{i}, t_{j}\right)
\end{aligned}
$$

and $\partial_{t}^{i, j}$ and $\partial_{x}^{i, j}$ are discretizations of the derivatives $\partial_{t}$ and $\partial_{x}$ respectively. A discrete version of the dissipation law can be written as follows

$$
\partial_{t}^{i, j} \omega_{i, j}+\partial_{x}^{i, j} \kappa_{i, j}=0
$$

where

$$
\omega_{i, j}=<M_{i, j} U_{i, j}, V_{i, j}>, \quad \kappa_{i, j}=<K_{i, j} U_{i, j}, V_{i, j}>
$$

Definition 3.1. The numerical scheme determined by equation (refds3.5) is called a multi-symplectic integrator if discrete law (21) is a dissipation law for equations (20).

\section{4 example}

We consider the following equation

$$
u_{t t}-u_{x x}+u+2 u_{t}=0
$$

it has a multi-symplectic Birkhoffian representation that has structure

$$
M=\left(\begin{array}{ccc}
0 & \mathrm{e}^{2 t} & 0 \\
-\mathrm{e}^{2 t} & 0 & 0 \\
0 & 0 & 0
\end{array}\right), \quad K=\left(\begin{array}{ccc}
0 & 0 & -\mathrm{e}^{2 t} \\
0 & 0 & 0 \\
\mathrm{e}^{2 t} & 0 & 0
\end{array}\right)
$$

and

$$
-\frac{1}{2} \mathrm{e}^{2 t}\left[u^{2}+p^{2}-q^{2}+2 u p\right]
$$

It has a dissipation law

$$
\frac{\mathrm{d}}{\mathrm{d} t}(\mathrm{~d} p \wedge \mathrm{d} u)+\frac{\mathrm{d}}{\mathrm{d} x}(\mathrm{~d} q \wedge \mathrm{d} u)=-2 \mathrm{~d} p \wedge \mathrm{d} u
$$


using the conditions of self-adjointness, the dissipation law can be rewritten as

$$
\operatorname{fracdd} t\left(\mathrm{e}^{2 t} \mathrm{~d} p \wedge \mathrm{d} u\right)+\frac{\mathrm{d}}{\mathrm{d} x}\left(\mathrm{e}^{2 t} \mathrm{~d} q \wedge \mathrm{d} u\right)=0 .
$$

Approximating the equation (22) in Birkhoffian structure as follows

$$
\begin{aligned}
\frac{\mathrm{e}^{\delta t} p_{i+1 / 2, j+1}-\mathrm{e}^{-\delta t} p_{i+1 / 2, j}}{\delta t}+\frac{q_{i+1, j+1 / 2}-q_{i, j+1 / 2}}{\delta x}= & -u_{i+1 / 2, j+1 / 2}, \\
\frac{u_{i+1 / 2, j+1}-u_{i+1 / 2, j}}{\delta t} & =p_{i+1 / 2, j+1 / 2}, \\
\frac{u_{i+1, j+1 / 2}-u_{i, j+1 / 2}}{\delta t} & =q_{i+1 / 2, j+1 / 2},
\end{aligned}
$$

where

$$
\begin{array}{r}
p_{i+1 / 2, j+1 / 2}=\frac{1}{4} \mathrm{e}^{\delta t}\left(p_{i+1, j+1}+p_{i, j+1}\right)+\frac{1}{4} \mathrm{e}^{-\delta t}\left(p_{i+1, j}+p_{i, j}\right), \\
u_{i+1 / 2, j+1 / 2}=\frac{1}{4}\left(u_{i+1, j+1}+u_{i, j+1}+u_{i+1, j}+u_{i, j}\right), \\
q_{i+1 / 2, j+1 / 2}=\frac{1}{4}\left(u_{i+1, j+1}+u_{i, j+1}+u_{i+1, j}+u_{i, j}\right) .
\end{array}
$$

Discrete the dissipation law (23) directly by using the same scheme, we get

$$
\begin{aligned}
& \frac{\mathrm{e}^{\delta t} \mathrm{~d} p_{i+1 / 2, j+1} \wedge \mathrm{d} u_{i+1 / 2, j+1}-\mathrm{e}^{-\delta t} \mathrm{~d} p_{i+1 / 2, j} \wedge \mathrm{d} u_{i+1 / 2, j}}{\delta t} \\
& -\frac{\mathrm{d} q_{i+1, j+1 / 2} \wedge \mathrm{d} u_{i+1, j+1 / 2}-\mathrm{d} q_{i, j+1 / 2} \wedge \mathrm{d} u_{i, j+1 / 2}}{\delta x}=0 .
\end{aligned}
$$

From the discrete equations (25), we also can construct the dissipation, i.e. (29) is a symplectic dissipation law for equations (25). So the scheme determined by equations (25) is a multi-symplectic integrator for Birkhoff's equations of system (22).

\section{References}

[1] T. J. Bridges \& S. Reich, Multi-symplectic integrators: numerical schemes for Hamiltonian PDEs that conserve symplecticity, Phys Lett A (in pres, 2001).

[2] R. M. Santilli, Foundations of Theoretical Mechanics I, 1983 by Springer-Verlag New York Inc.. 